\title{
New types of stable nonlinear whistler waveguides
}

\author{
Yu. A. Zaliznyak, T. A. Davydova, and A. I. Yakimenko \\ Plasma Theory Department, Institute for Nuclear Research, Nauki Ave. 47, Kiev 03680, Ukraine
}

Received: 7 June 2001 - Accepted: 28 August 2001

\begin{abstract}
The stationary self-focusing of whistler waves with frequencies near half of the electron-cyclotron frequency in the ionospheric plasma is considered in the framework of a two-dimensional generalized nonlinear Schrödinger equation including fourth-order dispersion effects and nonlinearity saturation. New types of solitonlike (with zero topological charge) and vortex-like nonlinear waveguides are found, and their stability confirmed both analytically and numerically.
\end{abstract}

\section{Introduction}

Plane whistler waves which propagate along the magnetic field $\boldsymbol{B}=\left(0,0, B_{z}\right)$ and obey the dispersion relation $k^{2} c^{2} / \omega_{p e}^{2}=\omega /\left(\omega_{B e}-\omega\right)$, are fully electromagnetic righthand polarized waves having $k_{\perp}=0, E_{z}=0$. However, when the wave beam is localized in the transverse plane, or when the direction of propagation deviates from the $O Z$ axis, these properties disappear. Together with nonzero $k_{\perp}$ whistler waves gain elliptic polarization (which can be assumed as a presence of the left-hand polarized part of the wave field) and the electrostatic wave component $\left(E_{z} \neq 0\right)$. Here we consider propagation of beams of whistler waves with frequencies near half of the electron-cyclotron frequency $\left(0<\omega_{B e} /(2 \omega)-1 \ll 1\right)$ strictly along the magnetic field in the ionosphere. Using a hydrodynamic approximation for cold plasma one can find that the electrostatic component $E_{z}(x, y)$ of the electrical field of the whistler wave in a linear case is governed by the following equation

$$
\begin{gathered}
\left\{\Delta_{\perp}^{2}+\left[-k_{z}^{2}-\frac{\varepsilon_{\|}}{\varepsilon_{\perp}} k_{z}^{2}+\frac{\omega^{2}}{c^{2}}\left(\varepsilon_{\|}+\varepsilon_{\perp}\right)-\frac{\omega^{2}}{c^{2}} \frac{g^{2}}{\varepsilon_{\perp}}\right] \Delta_{\perp}\right. \\
\left.+\frac{\varepsilon_{\|}}{\varepsilon_{\perp}}\left[\left(k_{x}^{2}-\frac{\omega^{2}}{c^{2}} \varepsilon_{\perp}\right)^{2}-\frac{\omega^{4}}{c^{4}} g^{2}\right]\right\} E_{z}=0,
\end{gathered}
$$

Correspondence to: Yu. A. Zaliznyak (zalik@kinr.kiev.ua) where $g=\left(\omega / \omega_{B e}\right) \omega_{p e}^{2} /\left(\omega^{2}-\omega_{B e}^{2}\right), \varepsilon_{\perp}=-\omega_{p e}^{2} /\left(\omega^{2}-\right.$ $\left.\omega_{B e}^{2}\right), \varepsilon_{\|}=-\omega_{p e}^{2} / \omega^{2}$. Taking into account the nonlinear frequency shift (see Gyrevich and Shvarzburg (1973))

$\omega_{n l}=-\frac{\omega^{2}+v_{e}^{2}}{2 v_{e}^{2}}\left(\sqrt{1+\frac{4 v_{e}^{2}}{\omega^{2}+v_{e}^{2}}\left|\frac{E_{z}}{E_{p}}\right|^{2}}-1\right)$

and expanding Eq. (2) accurately to $\sim\left|E_{z} / E_{p}\right|^{4}$, where $\omega \approx \omega_{B e} / 2, E_{p}=\sqrt{3 m T\left(\omega^{2}+v_{e}^{2}\right) \delta} / e^{2}, \delta \approx 2 m / M, v_{e}$ is the frequency of electron collisions, $n_{0}$ is the density of electrons, $T$ is the electron temperature, we obtain the Generalized Nonlinear Schrödinger Equation (GNSE):

$\mathrm{i} \frac{\partial \psi}{\partial \zeta}+D \Delta_{\perp} \psi+P \Delta_{\perp}^{2} \psi+B \psi|\psi|^{2}+K \psi|\psi|^{4}=0$,

where $\Delta_{\perp}=\partial^{2} / \partial \xi^{2}+\partial^{2} / \partial \eta^{2}, \psi=E_{z} / F_{0}, \delta \approx 2 m / M$, $F_{0}=\sqrt{12 \pi T n_{0} \delta}, \zeta=z \omega / c, \xi=x \omega / c, \eta=y \omega / c$, $\omega \approx \omega_{B e} / 2$, and the coefficients of equation are: $D=$ $\left(1 /\left(4 u^{2}\right)-1\right) /(2 \sqrt{v}), P=1 /\left(8 v^{3 / 2}\right), B=-v^{3 / 2}, K=$ $v^{5 / 2} v_{e}^{2} / \omega^{2}, u=\omega / \omega_{B e}, v=\omega_{P e}^{2} / \omega^{2}$. Equation (3) generalizes Eq. (1) of Zharova and Sergeev (1989) to the case of 2D geometry and takes into account the nonlinearity saturation effects. Gyrevich and Shvarzburg (1973) and Bharuthram et al. (1992) pointed out that the main nonlinear effect in the ionosphere is the plasma extrusion from the HF field region due to heating and a pressure increase. Near the point $\omega \approx \omega_{B e} / 2$ the thresholds of modulational instabilities for a whistler wave decreases dramatically, and one must account for the next terms in the nonlinearity expansion which are of the same order as the linear terms. It leads to the appearance of cubic-quintic saturable nonlinearity in the GNSE (3). In this paper we consider the case $u \leq 1 / 2$, which means $\omega \leq \omega_{B e} / 2$ and the signs of GNSE coefficients are the following: $D>0, P>0, B<0, K>0$. We are interested to find the conditions of the whistler wave propagation inside stationary waveguides, or channels, formed due to nonlinear self-interaction and localization in the plane perpendicular to direction of propagation: $O Z$ axis. Wave intensity profiles across these waveguides does not depend on $z$. 


\section{General properties of nonlinear whistler waveguides}

Any wave packet localized in the plane perpendicular to $z$ direction which evolves along the $z$-direction according to Eq. (3) has conserved quantities:

number of quanta:

$N=\iint|\psi|^{2} d^{2} \boldsymbol{r}$

momentum:

$\boldsymbol{I}=-\frac{\mathrm{i}}{2} \iint\left(\psi^{*} \nabla \psi-\psi \nabla \psi^{*}\right) d^{2} \boldsymbol{r}$,

angular momentum:

$\boldsymbol{M}=-\frac{\mathrm{i}}{2} \iint\left(\psi^{*}[\boldsymbol{r} \times \nabla \psi]-\psi\left[\boldsymbol{r} \times \nabla \psi^{*}\right]\right) d^{2} \boldsymbol{r}$,

Hamiltonian:

$$
\begin{gathered}
H=\iint\left(D|\nabla \psi|^{2}-P\left|\Delta_{\perp} \psi\right|^{2}-\right. \\
\left.\frac{B}{2}|\psi|^{4}-\frac{K}{3}|\psi|^{6}\right) d^{2} \boldsymbol{r} .
\end{gathered}
$$

Stationary (along the $O Z$ axis) waveguides in the framework of Eq. (3) have the form $\psi(\boldsymbol{r}) \exp (\mathrm{i} \lambda \zeta)$, where the complex function $\psi(\boldsymbol{r})$ obeys the partial differential equation

$$
-\lambda \psi+D \Delta_{\perp} \psi+P \Delta_{\perp}^{2} \psi+B \psi|\psi|^{2}+K \psi|\psi|^{4}=0 .
$$

Multiplying Eq. (8) by $\psi^{*}$ and integrating over space coordinates in the perpendicular plane, an integral relation

$$
\begin{aligned}
\lambda N & =P \iint\left|\Delta_{\perp} \psi\right|^{2} d^{2} \boldsymbol{r}-D \iint|\nabla \psi|^{2} d^{2} \boldsymbol{r} \\
& +B \iint|\psi|^{4} d^{2} \boldsymbol{r}+K \iint|\psi|^{6} d^{2} \boldsymbol{r}
\end{aligned}
$$

is obtained. Multiplying Eq. (8) by $r^{2} d \psi^{*} / d r$, integrating and adding the complex conjugate, another integral identity is found:

$$
\begin{aligned}
& \lambda N=-P \iint\left|\Delta_{\perp} \psi\right|^{2} d^{2} \boldsymbol{r}+ \\
& \frac{B}{2} \iint|\psi|^{4} d^{2} \boldsymbol{r}+\frac{K}{3} \iint|\psi|^{6} d^{2} \boldsymbol{r} .
\end{aligned}
$$

One restriction on the parameter $\lambda: \lambda<-D^{2} / 4 P$ easily follows from the asymptotic behavior $|r| \rightarrow \infty$ of any localized solution of Eq. (8). After excluding $\lambda$ from Eqs. (9)-(10) one finds a simplified expression for a Hamiltonian of solitary (which means decaying at infinity) solutions:

$$
\begin{aligned}
H & =P \iint\left|\Delta_{\perp} \psi\right|^{2} d^{2} \boldsymbol{r}+\frac{K}{3} \iint|\psi|^{6} d^{2} \boldsymbol{r} \\
& =\frac{D}{2} \iint|\nabla \psi|^{2} d^{2} \boldsymbol{r}-\frac{B}{4} \iint|\psi|^{4} d^{2} \boldsymbol{r} .
\end{aligned}
$$

From Eq. (11) it follows immediately that for $D>0, P>$ $0, B<0, K>0$ (which is the case under consideration) the Hamiltonian functional is always positive. Using integral inequalities

$\int|\nabla \psi|^{2} d^{2} \boldsymbol{r}<N^{1 / 2}\left(\int|\Delta \psi|^{2} d^{2} \boldsymbol{r}\right)^{1 / 2}$,

$\int|\psi|^{4} d^{2} \boldsymbol{r}<N^{1 / 2}\left(\int|\psi|^{6} d^{2} \boldsymbol{r}\right)^{1 / 2}$,

we find the following estimate for the Hamiltonian:

$$
\begin{aligned}
H< & D N^{1 / 2}\left(\int|\Delta \psi|^{2} d^{2} \boldsymbol{r}\right)^{1 / 2} \\
& -P \int|\Delta \psi|^{2} d^{2} \boldsymbol{r}+\frac{|B|}{2} N^{1 / 2}\left(\int|\psi|^{6} d^{2} \boldsymbol{r}\right)^{1 / 2} \\
& -\frac{K}{3} \int|\psi|^{6} d^{2} \boldsymbol{r}<\frac{N}{4}\left[\frac{D^{2}}{P}+\frac{3}{4} \frac{B^{2}}{K}\right] .
\end{aligned}
$$

It indicates that for a fixed value of the number of quanta $N$, a Hamiltonian functional is bounded from below and above. This guarantees that for any $N$ there exists at least one stable solitary solution which corresponds to Hamiltonian's absolute extremum.

Using integral inequalities Eqs. (12) and (13) and the identity Eq. (9) it is easy to show that the waveguide parameter $\lambda$ (the nonlinear shift of wave number) is bounded from below and to estimate a range of accessible $\lambda$ - values:

$-\frac{D^{2}}{4 P}-\frac{B^{2}}{4 K}<\lambda<-\frac{D^{2}}{4 P}$.

From the virial relation for the waveguide's effective width $r_{\mathrm{eff}}^{2}=N^{-1} \int_{0}^{2 \pi} \int_{0}^{\infty} r^{2}|\psi|^{2} r d r d \theta$,

namely, from

$$
\begin{aligned}
& N \frac{d^{2} r_{\mathrm{eff}}^{2}}{d \zeta^{2}}=\int_{0}^{\infty} \int_{0}^{2 \pi}\left\{8 \left[D^{2}|\nabla \psi|^{2}-4 D P|\Delta \psi|^{2}\right.\right. \\
& \left.\quad+4 P^{2}|\nabla \Delta \psi|^{2}\right]-\frac{B D}{2}|\psi|^{4} \\
& \quad-\frac{K D}{3}|\psi|^{6}-3 B \operatorname{Pr}|\nabla \psi|^{2}\left(\nabla|\psi|^{2}\right) \\
& \left.-P K r\left(\nabla|\psi|^{2}\right)\left[6|\psi|^{2}|\nabla \psi|^{2}+\left(\nabla|\psi|^{2}\right)^{2}\right]\right\} r d r d \theta,(16)
\end{aligned}
$$

one finds that: (i) when $P=K=0$ and $B D<0$, GNSE (3) has no localized solutions at all; any wave packet moving along the $z$-axis spreads out in a radial direction; (ii) in the case of $P=0=K=0, B D>0$ the virial relation gives

$N \frac{d^{2} r_{e f f}^{2}}{d \varsigma^{2}}=8 D H$,

and predicts the collapsing of any wave packet having DH $<0$; (iii) the sum of all linear terms in the virial relation 
are defocusing. This follows from the integral inequality

$$
\begin{aligned}
& \int_{0}^{\infty}\left|\Delta_{r} \psi\right|^{2} r d r=-\int_{0}^{\infty} \frac{d \psi}{d r}\left(\frac{d \Delta_{r} \psi}{d r}\right) r d r \leq \\
& \left(\int_{0}^{\infty}\left|\frac{d \psi}{d r}\right|^{2} r d r\right)^{1 / 2}\left(\int_{0}^{\infty}\left|\frac{d \Delta_{r} \psi}{d r}\right|^{2} r d r\right)^{1 / 2} .
\end{aligned}
$$

Thus, any linear wave packet described by the linear GNSE will always spread out. When $D>0, B<0, P>0, K>0$ the virial relation includes focusing (proportional to $K D$ and to $B P$ ) as well as defocusing (proportional to $B D$ and $P K$ ) nonlinear terms. Different linear and nonlinear terms come into play at different spatial scales and on different values of field amplitude; thus it is natural to expect that several stationary nonlinear structures may coexist in the framework of GNSE (3).

\section{Variational analysis}

In the framework of the variational approach (see Anderson, 1983) the GNSE (3) is formulated as a reduced variational problem for functional $\int L d \zeta$

$\delta \int L d \zeta=0$

where $L=\int l d^{2} r, l$ is the Lagrangian density. In cylindrical coordinates $(r, \theta, \zeta)$ the latter may be written as:

$$
\begin{aligned}
l= & \frac{i}{2}\left[\psi \frac{\partial \psi^{*}}{\partial \zeta}-\psi^{*} \frac{\partial \psi}{\partial \zeta}\right]+D\left[\left|\frac{\partial \psi}{\partial r}\right|^{2}+\frac{1}{r^{2}}\left|\frac{\partial \psi}{\partial \theta}\right|^{2}\right] \\
& -P\left|\frac{1}{r} \frac{\partial}{\partial r}\left(r \frac{\partial \psi}{\partial r}\right)+\frac{1}{r^{2}} \frac{\partial^{2} \psi}{\partial \theta^{2}}\right|^{2}-\frac{B}{2}|\psi|^{4}-\frac{K}{3}|\psi|^{6}
\end{aligned}
$$

Here we will use a trial function in the form:

$$
\psi=\frac{\mu \sqrt{N}}{\sqrt{2 \pi I_{H}^{(l)}}} f_{l}(\mu r) \exp [i \gamma \ln \cosh \mu r+i l \theta+i \varphi(\zeta)]
$$

where $\mu=\mu(\zeta), \gamma=\gamma(\zeta)$, and the real function $f_{l}(\xi)$ determines the envelope profile:

$f_{l}(\xi)=\frac{\xi^{|l|}}{\cosh (\xi)}$.

This function describes soliton-like (at $l=0$ ) or vortexlike (at $l \neq 0$ ) nonlinear waveguides. The nonlinear phase dependence on $r$-coordinate for this function is fixed to be $\sim i \gamma \ln \cosh \mu r$, as it was in the case of 1D exact chirped soliton solution found by Davydova and Zaliznyak (2000). After performing a Ritz optimization procedure, the waveguide parameters $\mu$ and $\beta=\gamma \mu$ are obtained from the canonical Hamiltonian set of equations

$$
\begin{aligned}
& \frac{d \beta}{d \eta}=\frac{\partial H}{\partial \mu} \\
& \frac{d \mu}{d \eta}=-\frac{\partial H}{\partial \beta}
\end{aligned}
$$

where $\eta=\frac{1}{N} \int_{0}^{z} \mu^{2} d \zeta$, and the Hamiltonian

$$
\begin{aligned}
H= & \frac{N}{2 \pi I_{H}^{(l)}}\left[D\left(I_{d 1}^{(l)} \mu^{2}+I_{d 2}^{(l)} \beta^{2}\right)-\frac{B I_{b}^{(l)}}{2} N \mu^{2}\right. \\
& \left.-\frac{K I_{k}^{(l)}}{3} N^{2} \mu^{4}-P\left(I_{p 1}^{(l)} \mu^{4}+I_{p 2}^{(l)} \mu^{2} \beta^{2}+I_{p 3}^{(l)} \beta^{4}\right)\right] .
\end{aligned}
$$

Integrals $I_{\sigma}^{(l)}$ are defined by the choice of trial function:

$$
\begin{aligned}
I_{d 1}^{(l)} & =\int_{0}^{\infty} \xi\left(\frac{\partial f_{l}(\xi)}{\partial \xi}\right)^{2} d \xi ; \\
I_{d 2}^{(l)} & =\int_{0}^{\infty} \xi f_{l}^{2}(\xi) \tanh ^{2}(\xi) d \xi ; \\
I_{b}^{(l)} & =\frac{1}{2 \pi I_{H}^{(l)}} \int_{0}^{\infty} \xi f_{l}^{4}(\xi) d \xi ; \quad I_{H}^{(l)}=\int_{0}^{\infty} \xi f_{l}^{2}(\xi) d \xi \\
I_{p 1}^{(l)} & =\int_{0}^{\infty} f_{l}^{2}(\xi)\left(1-\frac{2}{(\cosh \xi)^{2}}-\frac{(2 l+1) \tanh \xi}{\xi}\right)^{2} d \xi \\
I_{p 3}^{(l)} & =\int_{0}^{\infty} \xi \tanh ^{4} \xi f_{l}^{2}(\xi) d \xi ; \quad I_{p 2}^{(l)}=I_{p 1}^{(l)}+I_{p 3}^{(l)} ; \\
I_{k}^{(l)} & =\frac{1}{\left(2 \pi I_{H}^{(l)}\right)^{2}} \int_{0}^{\infty} \xi f_{l}^{6}(\xi) d \xi .
\end{aligned}
$$

Stationary points of the system Eq. (21) coincide with the extrema of the Hamiltonian functional Eq. (22):

$\left.\frac{\partial H}{\partial \beta}\right|_{\mu=\mu_{0}, \beta=\beta_{0}}=0 ;\left.\quad \frac{\partial H}{\partial \mu}\right|_{\mu=\mu_{0}, \beta=\beta_{0}}=0$.

These points determine the waveguide parameters $\mu_{0}$ and $\beta_{0}$ for a given number of quanta $N$. Introducing the notation

$N_{1}=\frac{D I_{d}^{(l)}\left(I_{p 1}^{(l)}-I_{p 3}^{(l)}\right)}{|B| I_{b}^{(l)} I_{p 3}^{(l)}} ; \quad N_{2}^{2}=\frac{3 P\left(I_{p 1}^{(l)}-I_{p 3}^{(l)}\right)^{2}}{4 I_{p 3}^{(l)} K I_{k}^{(l)}}$,

$\sigma=\frac{4\left(D I_{d p}^{(l)}\right)^{2} K I_{k}^{(l)}}{3\left(B I_{b}^{(l)}\right)^{2} P I_{p 3}^{(l)}}=\left(\frac{N_{1}}{N_{2}}\right)^{2}$,

where

$I_{d p}^{(l)}=\frac{I_{p 1}^{(l)}-I_{p 3}^{(l)}\left(2 I_{d 1}^{(l)} / I_{d 2}^{(l)}-1\right)}{I_{p 1}^{(l)}-I_{p 3}^{(l)}}$,

one obtains for the waveguides with $\beta=0$ the following relation connecting $\mu^{2}$ and $N$ :

$\mu_{o}^{2}=\frac{D I_{d}^{(l)}+\frac{|B| I_{b}^{(l)}}{2} N}{2\left(P I_{p 1}^{(l)}+\frac{K I_{k}^{(l)}}{3} N^{2}\right)}<\mu_{o \text { max }}^{2}$,

where

$\mu_{o \max }^{2}=\frac{D I_{d}^{(l)}}{4 P I_{p 1}^{(l)}}\left(1+\sqrt{1+\frac{3\left(B I_{b}^{(l)}\right)^{2} P I_{p 3}^{(l)}}{4\left(D I_{d 1}^{(l)}\right)^{2} K I_{k}^{(l)}}}\right)$. 
One can also find stationary solutions of Eq. (24) with $\beta_{0} \neq$ 0 which correspond to electromagnetic beams with curved wave fronts. In particular for $l=0$ one has

$\mu_{c}^{2}=\frac{D I_{d}^{(0)}}{P\left(I_{p 1}^{(0)}-I_{p 3}^{(0)}\right)} \frac{1-N / N_{1}}{1-\left(N / N_{2}\right)^{2}}$,

$$
\begin{aligned}
\mu_{c}^{2}(N=0) & =\frac{D I_{d}^{(0)}}{P\left(I_{p 1}^{(0)}-I_{p 1}^{(0)}\right)}>\frac{D I_{d}^{(0)}}{P I_{p 2}^{(0)}}> \\
\frac{D I_{d}^{(0)}}{2 P I_{p 1}^{(0)}} & =\mu_{o}^{2}(N=0) .
\end{aligned}
$$

Some stationary solutions are stable under the variation of two parameters, $\beta$ and $\mu$, when they give maximum or minimum to the Hamiltonian and they are unstable when they correspond to the Hamiltonian saddle point. It gives a stability criterion in the framework of our variational approach in the form:

$h=\frac{\partial^{2} H}{\partial \beta^{2}} \frac{\partial^{2} H}{\partial \mu^{2}}-\left.\left(\frac{\partial^{2} H}{\partial \beta \partial \mu}\right)^{2}\right|_{\beta=\beta_{0}, \mu=\mu_{0}}>0$.

The qualitative dependencies of $N$ on $\mu_{0}^{2}$ for waveguides with $\beta_{0}=0, l=0$ (marked "O") and for $\beta_{0} \neq 0, l=0$ (marked "C") are plotted in the Fig. 1. The dashed line indicates the region where $\beta^{2}<0$ and thus where waveguides with $\beta \neq 0$ cannot exist. Stable and unstable branches are marked in this figure by "S" and "US", respectively. It is seen that the variational approach with a trial function Eq. (19) predicts a bistability phenomenon for stationary waveguide propagation states: the coexistence of two waveguides with the same width but with different numbers of quanta, see Fig. 1a in the region $\left(d / p_{2}, \mu_{o \text { max }}^{2}\right)$. The conclusion of bistability also will be confirmed by the direct numerical solution of the stationary and nonstationary equations in the next section.

\section{Numerical modeling}

In order to perform a numerical modeling of GNSE waveguides, it is more convenient to use it in a fully $3 \mathrm{D}$ form in Cartesian coordinates $(\xi, \eta, \zeta)$. This equation has no peculiarity at the point $r=0$. After the standart rescalings, this equation takes a form

$\mathrm{i} \frac{\partial \psi}{\partial \zeta}+\Delta_{\perp} \psi+\Delta_{\perp}^{2} \psi-\psi|\psi|^{2}+\kappa \psi|\psi|^{4}=0$,

where $\kappa=K D^{2} / P B^{2}$. Without loss of generality we shall assume below that $D=P=1, B=-1$ and vary parameter $K$. Stationary (along $O Z$ ) solutions have a form $\psi(\xi, \eta, \zeta)=\psi(\xi, \eta) \exp (\mathrm{i} \lambda \zeta)$ and obey the partial differential equation:

$-\lambda \psi+\Delta_{\perp} \psi+\Delta_{\perp}^{2} \psi-\psi|\psi|^{2}+\kappa \psi|\psi|^{4}=0$.

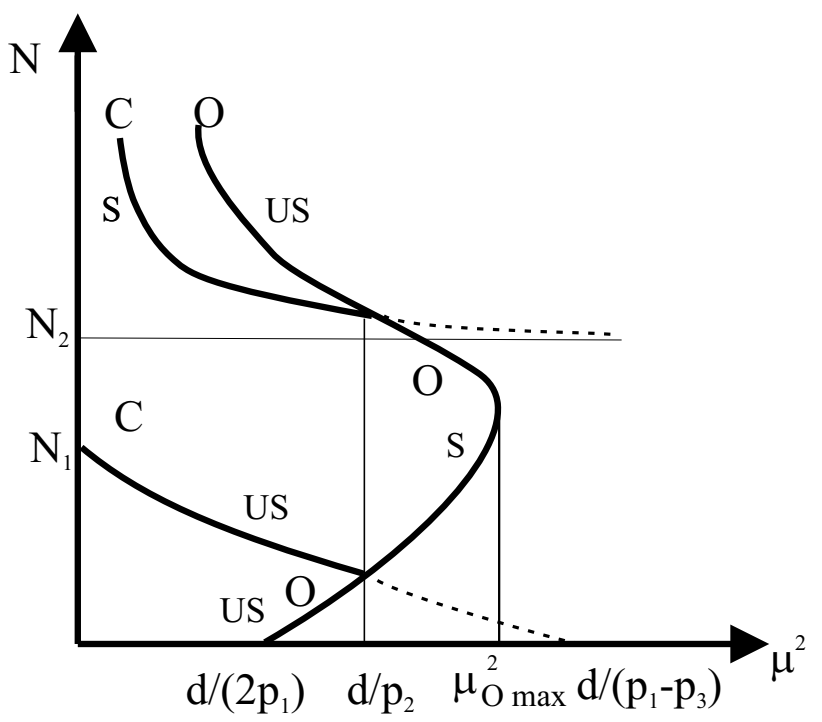

(A)

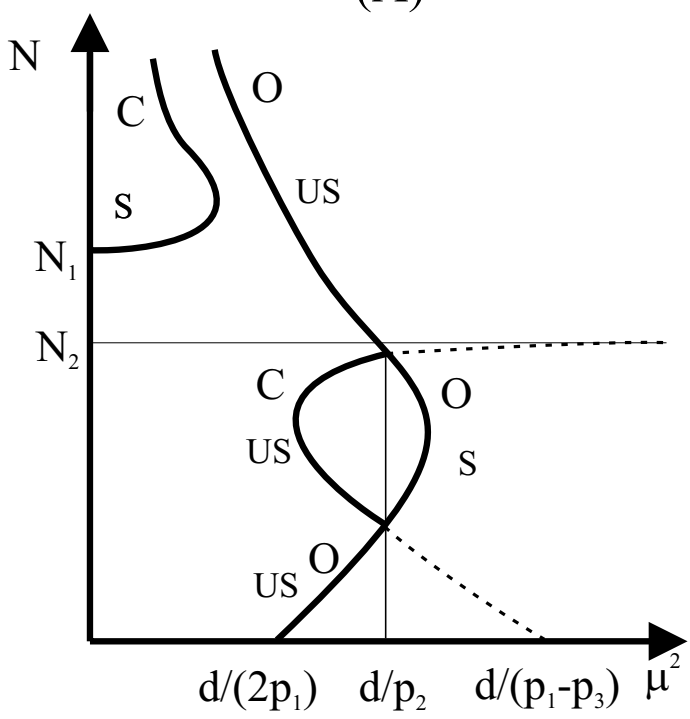

(B)

Fig. 1. $N$ versus $\mu^{2}, d=D I_{d}^{(l)}, p_{\sigma}=P I_{\sigma}^{(l)}$ (a): for the case when $N_{1}<N_{2}(\sigma<1)$.; (b): for the case when $N_{1}>N_{2}(\sigma>1)$.

The Eq. (33) was integrated numerically using the relaxation process in the Fourier space which is a generalization of a well-known stabilizing multiplier method, described in Petviashvili and Pokhotelov (1989). To check the structure's stability, the evolutionary Eq. (32) was also integrated numerically by a standart split step Fourier method (see Taha and Ablowitz, 1984).

Simulation results are presented in a form of energy dispersion diagrams (EDD) - dependencies $N(\lambda)$. EDD for soliton, like $(l=0)$ and vortex, like $(l=1)$ nonlinear structures with $\beta_{0}=0$ for different values of $\kappa$ are plotted in Fig. 2a and $b$.

It is seen that soliton like and vortex-like waveguides exist inside the restricted (both in $N$ and in $\lambda$ ) area and the size 


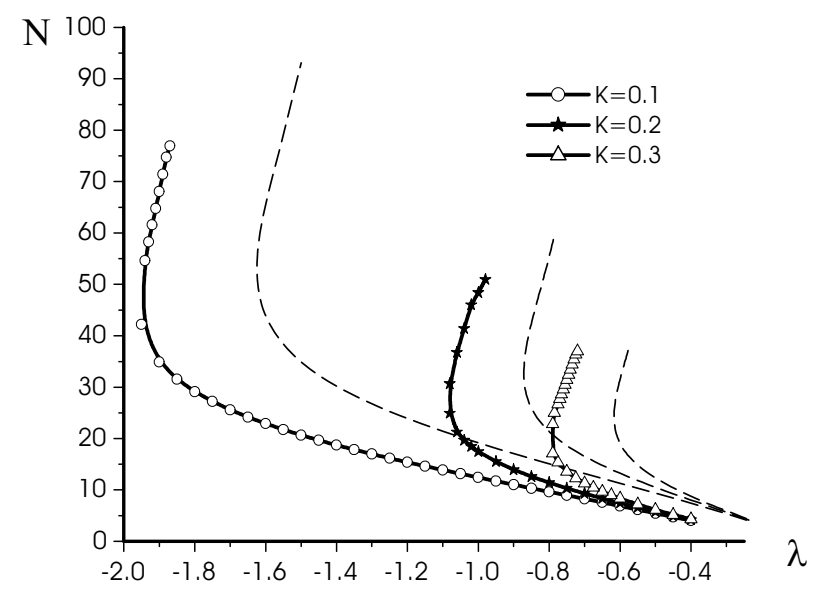

(A)

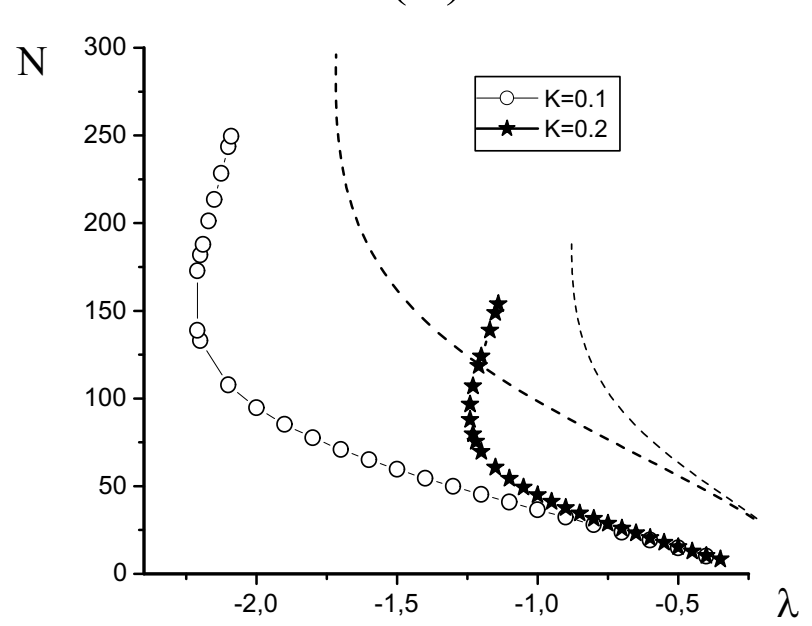

(B)

Fig. 2. An energy dispersion diagram for stationary soliton, like $(l=0$, case (a)) and vortex, like $(l=1$, case $(\mathbf{b}))$ solutions of GNSE. Numerical result. Dashed lines indicate $N(\lambda)$ dependencies predicted by the variational approach. Here $D=P=-B=1$.

of their existence domain decreases when $K$ increases. At every solitary or vortex branch there exists a range of $\lambda-$ values, where our numerical simulations confirm the variational conclusions about the bistability of stationary waveguide propagation states in the sense of the coexistence of two states with the same $\lambda$ but with different energies (numbers of quanta) and spatial scales. Examples of bistable soliton like and vortex like states are presented in Figs. 3a and b.

A numerical verification of stationary waveguide stability has shown that all waveguides found above with $l=0$ and $l=1$ are stable even with respect to sufficiently high symmetric and asymmetric perturbations of the initial state. Their parameters (width, amplitude, etc.) oscillate nonlinearly in the vicinity of the stationary point. A conclusion about the waveguide stability does not depend on the sign of $\partial N / \partial \lambda$ derivative in contrast to the well-known Kolokolov-
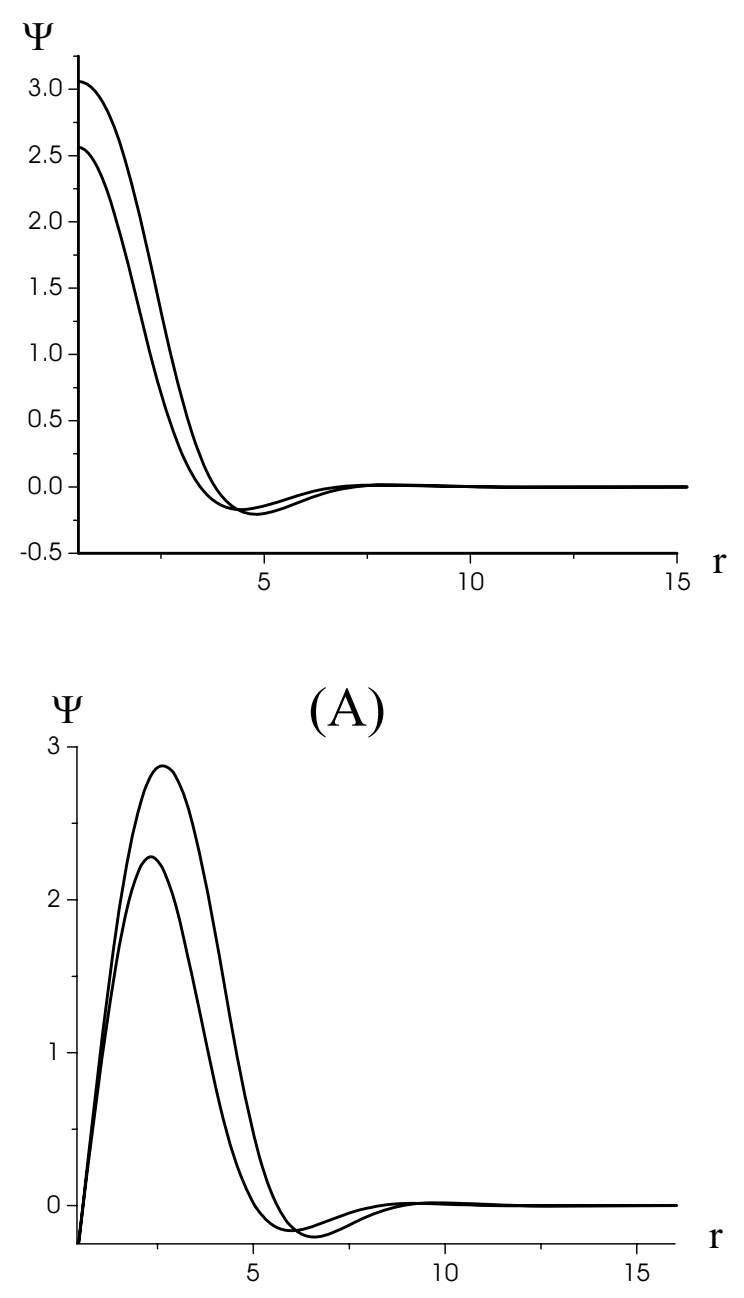

(B)

Fig. 3. Stable stationary radially, symmetric soliton, like $(l=0$, case (a), $\lambda=-1.89)$ and vortex, like $(l=1$, case $(\mathbf{b}), \lambda=-2.10)$ solutions of GNSE with $D=P=-B=1, K=0.1$.

Vakhitov criterion which takes place in the case of $P=0$. When the parameter $K$ exceeds some threshold value $K_{c r}$, localized solutions of Eq. (33) disappear.

It was also confirmed numerically that soliton parameter $\lambda$ is bounded from below: for every $K$ there exists some minimum value of $\lambda=\lambda_{\min }$ (see Fig. 4).

\section{Conclusions}

The propagation of whistler wave beams along the magnetic field lines with frequencies near the half electron gyrofrequency $\left(\omega \approx \omega_{B e} / 2\right)$ in the ionosphere is described by the single nonlinear Schrödinger equation for the parallel electric field component Eq. (3). This equation includes a term $\sim P \Delta_{\perp}^{2} E_{z}$ in its linear part. 


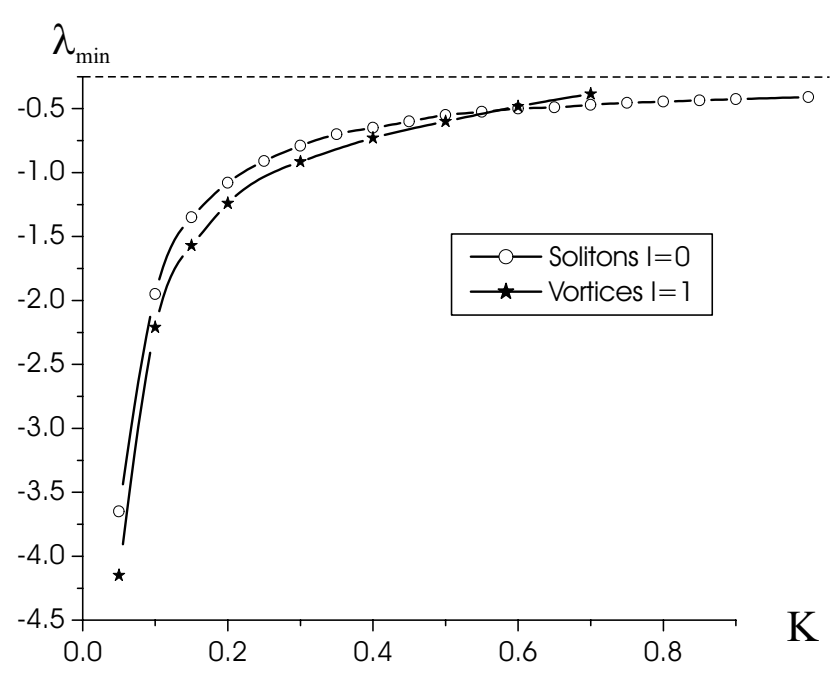

Fig. 4. The minimum possible value of $\lambda$ (where soliton, like $l=$ 0 and vortex, like $l=1$ waveguides exist) versus $K$. Numerical result. Here $D=P=-B=1$.

In the considered case $D>0, P>0, B<0, K>0$, the Hamiltonian of GNSE (3) is bounded from below and above for every $N$, which indicates that there exists at least one stable solitary solution, in the Lyapunov sense, which corresponds to the Hamiltonian's exact extremum.

The sum of all linear terms in the virial relation for the waveguide's effective width acts in a defocusing manner, which indicates that any linear wave packet described by a linear GNSE (3) always spreads out. At the same time the nonlinear part of the virial relation includes focusing, as well as defocusing terms. It may result in a coexistence of several stationary nonlinear structures with different spatial scales in the framework of Eq. (3).

A variational approach with the trial function Eq. (19) predicts a bistability phenomenon for the stationary waveguide propagation states: the coexistence of two stable solutions with different energies (numbers of quanta $N$ ) and spatial scales for the same value of the nonlinear shift of wave number $\lambda$. It also predicts that the sign of the derivative $\partial N / \partial \lambda$ may change within the region of stability, thus a commonly used Vakhitov-Kolokolov criterion (which works for GNSE with $P=0$ ) in the considered case $P>0, K>0$ cannot be applied.

Direct numerical integration of stationary (with respect to Z) (Eq. 8) and nonstationary (Eq. 3) equations showed that there really exists soliton, like (with zero topological charge) and vortex, like (first azimuthal mode $l=1$ ) stable nonlinear waveguides. Simulations found exactly the bistability regions for waveguides (see Fig. 2). All stationary solutions were shown to be stable even with respect to sufficiently high symmetric and asymmetric perturbations of the initial state.

The developed theory can be helpful for explaining the regular structures formed during ionospheric heating experiments (Bharuthram et al., 1992), e.g. for altitude $h=300 \mathrm{~km}$ that corresponds to maximum of the F-layer of the ionosphere: $n_{e} \approx 2 \cdot 10^{6} \mathrm{~cm}, T_{e} \approx 10^{3}{ }^{\circ} \mathrm{K}, \omega_{B e} \approx 8 \cdot 10^{6}$ $\mathrm{c}^{-1} . D \approx 2.5 \cdot 10^{-2} \cdot \Delta, P \approx 1.6 \cdot 10^{-5}, B \approx-8 \cdot 10^{3}$, $K \approx 0.8 v_{e}^{2}$ and $\kappa=\frac{K D^{2}}{P B^{2}} \approx 10^{-7} v_{e}^{2} \Delta^{2} \ll 1$, where $1 \gg \Delta=\left(\frac{\omega_{B e}}{2 \omega}\right)^{2}-1>0$. Thus typical space scales of the structures depend on $\Delta: L_{\perp}[\mathrm{cm}] \simeq 6 \cdot 10^{2} / \sqrt{\Delta}$, $L_{||}[\mathrm{cm}] \simeq 6 \cdot 10^{2} / \Delta^{2}$. We think that there is a possibility to observe such structures in ionosphere.

\section{References}

Anderson, D.: Variational approach to nonlinear pulse propagation in otptical fibers, Physical Review, A 27, 3135-3145, 1983.

Bharuthram, R., Shukla, P. K., Stenflo, L., Nekrasov, A. K., and Pokhotelov, O. A.: Generation of coherent structures caused by ionospheric heating, IEEE Trans. Plasma Sciense, 20, 6, 803809, 1992.

Davydova, T. A. and Zaliznyak, Yu. A.: Chirped Solitons Near Plasma Resonances in the Magnetized Dlasmas, Physica Scripta, 69, 476-484, 2000.

Gyrevich, A. V. and Shvarzburg, A. B.: Nonlinear theory of radiowave propagation in ionosphere (in Russian), Moskow, Nauka, 1973.

Petviashvili, V. I. and Pokhotelov, O. A.: Solitary waves in plasma and atmosphere (in Russian), Moscow, Energoatomizdat, 1989.

Taha, M. J. and Ablowitz, M. J.: Analytical and Numerical Aspects of Certain Nonlinear Evolution Equations. II. Numerical, Nonlinear Schrödinger Equation, J. Comput. Phys., 55, 203-230, 1984.

Zharova, N. A. and Sergeev, A. M.: On the stationary self-influence of whistler waves Fizika Plazmy, 15, 1175-1179, 1989. 